\title{
Mild Traumatic Brain Injury and Suicide Risk Among a Clinical Sample of Deployed Military Personnel: Evidence for a Serial Mediation Model of Anger and Depression
}

\author{
Ian H. Stanley, M.S.; ${ }^{\mathrm{a}}$ Thomas E. Joiner, Ph.D.; ${ }^{\mathrm{a}}$ Craig J. Bryan, Psy.D., ABPP, \\ a Department of Psychology, Florida State University, Tallahassee, Florida USA \\ bepartment of Psychology, University of Utah, Salt Lake City, Utah USA \\ ${ }^{\mathrm{c}}$ National Center for Veterans Studies, Salt Lake City, Utah USA
}

Corresponding Author:

Ian H. Stanley

Department of Psychology

Florida State University

1107 West Call Street

Tallahassee, FL 32306-4301

stanley@psy.fsu.edu 


\begin{abstract}
Research has demonstrated a robust link between traumatic brain injuries (TBIs) and suicide risk. Yet, few studies have investigated factors that account for this link. Utilizing a clinical sample of deployed military personnel, this study aimed to examine a serial meditation model of anger and depression in the association of mild TBI and suicide risk. A total of 149 military service members referred for evaluation/treatment of a suspected head injury at a military hospital participated in the present study (92.6\% male; $\left.M_{\mathrm{age}}=27.9 \mathrm{y}\right)$. Self-report measures included the Suicidal Behaviors Questionnaire-Revised (SBQ-R), Automated Neuropsychological Assessment Metrics (ANAM) anger and depression subscales, and Behavioral Health Measure-20 depression subscale. A current mild TBI diagnosis was confirmed by a licensed clinical psychologist/physician. Overall, $84.6 \%(126 / 149)$ of participants met diagnostic criteria for a current mild TBI. Bootstrapped serial mediation analyses indicated that the association of mild TBI and suicide risk is serially mediated by anger and depression symptoms (bias-corrected 95\% confidence interval $[\mathrm{CI}]$ for the indirect effect $=0.044,0.576$ ). An alternate serial mediation model in which depression symptoms precede anger was not statistically significant (bias-corrected $95 \% \mathrm{CI}$ for the indirect effect $=-0.405,0.050$ ). Among a clinical sample of military personnel, increased anger and depression statistically mediated the association of mild TBI and suicide risk, and anger appears to precede depression in this pathway. Findings suggest that therapeutically targeting anger may serve to thwart the trajectory to suicide risk among military personnel who experience a mild TBI. Future research should investigate this conjecture within a prospective design to establish temporality.
\end{abstract}

Key Words: TBI; suicide; suicidality; anger; depression 


\section{Mild Traumatic Brain Injury and Suicide Risk Among a Clinical Sample of Deployed Military Personnel: Evidence for a Serial Mediation Model of Anger and Depression}

Suicide is the tenth leading cause of death in the general U.S. adult population (Centers for Disease Control and Prevention [CDC], 2016), and the second leading cause of death among military personnel, in particular (Ramchand et al., 2011). The increased risk observed among military personnel has been intractable across the past several years, with rates steadily increasing since as early as 2001 (Hoge and Castro, 2012; Kuehn, 2009; Ramchand et al., 2011). As such, the U.S. Department of Defense (Defense Suicide Prevention Office, 2014), the U.S. Department of Veterans Affairs (Kemp and Bossarte, 2013), and the U.S. Surgeon General (2012) have identified the understanding and prevention of suicide among military personnel as a critical public health priority.

To address the rising suicide rates within the military, it is essential to consider suicide risk factors that may be unique to this population. For one, military training- and combat-related exposures, such as explosions and falls, may result in concussive injuries that meet diagnostic threshold for a traumatic brain injury (TBI). A burgeoning area of research has demonstrated that TBIs are associated with increased risk for suicidal ideation, suicide attempts, and death by suicide across both veteran (Brenner et al., 2011; Gradus et al., 2015; Gutierrez et al., 2008) and some (Bryan and Clemans, 2013) but not all (Skopp et al., 2012) active duty samples (see Bahraini, Simpson, Brenner, Hoffberg, \& Schneider, 2013 for review). The association between TBI and suicide risk appears to persist across TBI severity levels, with a study of TBI patients in Denmark demonstrating standardized mortality ratios of 3.0, 2.7, and 4.1 for mild, moderate, and severe TBIs, respectively (Teasdale and Engberg, 2001). That TBIs are associated with a marked increase in suicide risk is especially concerning, given that between $19.5 \%$ and $45 \%$ of military 
service members and veterans have experienced a probable TBI (Brenner et al., 2013; Tanielian and Jaycox, 2008).

Despite the growing evidence base linking TBIs with increased suicide risk, it remains largely unknown what factors may account for this link. As such, researchers have recently encouraged investigations into pathways accounting for the link between TBI and suicide risk (Bryan and Clemans, 2013). Although TBI symptoms span both physical (e.g., headaches) and psychological (e.g., irritability) domains, evidence suggests that TBI-related psychological symptoms are more likely to persist post-injury (Terrio et al., 2009) and have greater relevance to suicide-related outcomes. Past research has revealed that comorbid conditions and psychological consequences of a TBI include substance abuse, posttraumatic stress disorder (PTSD), apathy, and depression (Stéfan and Mathé, 2016). One of the most prevalent psychiatric diagnoses following a TBI is major depressive disorder (Koponen et al., 2002), and preliminary evidence suggests that among U.S. veterans, depression, but not PTSD symptoms, may account for the link between TBI and suicidal ideation (Gradus et al., 2015).

One chief psychological consequence of a TBI that has received relatively sparse empirical attention with regard to suicidality is anger. Among a large sample of military personnel with $(\mathrm{N}=661)$ and without $(\mathrm{N}=1,024)$ a history of $\mathrm{TBI}$, Bailie et al. (2015) found that TBI is associated with increased problems with the experience, expression, and control of anger; in this study, $93.3 \%$ of the clinical subsample and $91.4 \%$ of the nonclinical subsample had a mild TBI diagnosis. The construct of anger is hypothesized to consist of a spectrum of subdomains, such as irritability, aggression, and hostility (Potegal and Stemmler, 2010). Although a relatively robust body of literature has examined TBI and these subdomains (Alderman, 2003; Kim et al., 1999; Rao et al., 2009), few studies have examined the impact of TBI on the broad spectrum of 
anger (Bailie et al., 2015), and only one study of which we are aware has investigated TBI, anger, and suicidality among military personnel. That is, Brenner et al. (2015) examined the State-Trait Anger Expression Inventory-2 (STAXI-2) among a sample of 133 military veterans with and without a history of a moderate-to-severe TBI and suicide attempts. There was no statistically significant between-group difference in STAXI-2 scores, although veterans with a suicide attempt history and a TBI had an approximately $25 \%$ higher anger expression index score than veterans with a suicide attempt history and no TBI as well as veterans with a TBI and no history of a suicide attempt. Thus, this initial finding suggests that anger may be a key factor in the association of TBI and suicide risk.

However, a single study does not provide sufficient insight into the role of anger in the association of TBI and suicide risk; replication across distinct samples is needed. Moreover, it is imperative that research examines the interplay of TBI, anger, and suicide risk within a mediation model, in order to classify anger as a possible mechanism amenable to therapeutic intervention. Given the dearth of research in this domain, it is also important to examine the broad construct of anger (encompassing irritability, aggression, and hostility; Potegal \& Stemmler, 2010) in order to parse apart this heterogeneous construct in future research.

The psychological consequences of TBIs noted above, including anger and depression, also have established relevance to suicide risk. Namely, a constellation of risk factors for suicide has been identified that generally includes two categories - overarousal states (e.g., anger) and underarousal states (e.g., depression) — and although these two categories seemingly represent polarities, serious suicidal crises are characterized by the simultaneous presence of overarousal and underarousal states (Chu et al., 2015; Joiner and Stanley, in press). Data from a nationallyrepresentative study in the U.S. indicate that anger experience and expression is associated with 
increased rates of suicide ideation, plans, and attempts; moreover, anger uniquely predicts who among suicide ideators have made a suicide attempt (Hawkins and Cougle, 2013). Data from psychiatric outpatients suggest that perceived burdensomeness-a core component of the interpersonal theory of suicide (Joiner, 2005; Van Orden et al., 2010), in which one believes that one's death is worth more than one's life to others - accounts for the link between anger and suicidal ideation. The interpersonal theory of suicide implicates perceived burdensomeness in the pathogenesis of suicidal desire; however, other variables are crucial to the understanding of suicide attempts (Van Orden et al., 2010). Regarding anger and suicide among military populations, past research of nondeployed soldiers indicates that intermittent explosive disorder (IED), a disorder largely characterized by problematic anger (American Psychiatric Association, 2013), predicts suicide attempts among suicide ideators (Nock et al., 2014).

It is, therefore, crucial to further elucidate the ways by which anger contributes to suicidality, including through its relation to other psychiatric conditions, such as depression. A substantial empirical base has identified depression as a risk factor for suicide (Brown et al., 2000; Minkoff et al., 1973); psychological autopsy studies suggest that most suicide decedents have a depressive disorder at the time of death (Cavanagh et al., 2003). Separate research has suggested that, although distinct constructs, anger and depression are highly interrelated (Fava et al., 1993; Luutonen, 2007), with some studies suggesting that anger is specific to depressive as compared to anxiety disorders (Koh et al., 2002).

\section{The Present Study}

The primary aim of this study was to determine if the relationship between mild TBI and suicide risk is statistically accounted for by anger and depression symptoms among a clinical sample of military personnel referred for evaluation/treatment of suspected head injury at a TBI 
clinic within a military combat support hospital in Iraq. We hypothesized that the indirect effects of anger and depression symptoms would significantly account for the relationship between mild TBI and suicide risk. We also hypothesized that the order of the variables would matter, such that anger precedes depression in its association of mild TBI and suicide risk in a serial mediation model. To our knowledge, this is the first study examining anger, particularly as it serves as a precursor to depression symptoms, as a mediator potentially accounting for the link between mild TBI and suicidality. Although ideally an investigation into the pathways by which a mild TBI increases suicide risk among military personnel would employ a prospective design, such an undertaking would require substantial financial, administrative, and human participant resources.

\section{Materials and Methods}

\section{Participants}

Participants included 147 military personnel and 2 civilian contractors $(\mathrm{N}=149)$ who were referred to a TBI clinic within a military hospital in Iraq during a 6-month span in 2009 for evaluation/treatment of a suspected head injury. Patients were predominately male (92.6\%) and white $(71.4 \%)$. Respondents had an average of $6.7(\mathrm{SD}=5.4)$ years in the military and were deployed between 0 and 6 times (mean=0.8, SD=1.1); the majority was in the Army (79.9\%) and junior enlisted (rank E1-E4; 55.8\%). See Table 1 for additional participant characteristics.

\section{Procedures}

Military personnel with a suspected brain injury were referred to an outpatient TBI clinic either directly from a treating medical provider or from the battlefield (Bryan and Clemans, 2013). Patients who provided written informed consent for research participation underwent a standardized evaluation, which included computerized neurocognitive testing, a battery of 
psychological and physical health questionnaires, and a clinical interview conducted by a clinical psychologist or physician who had advanced training in the assessment, diagnosis, and clinical management of TBI. Patients who had sustained a moderate to severe TBI and who were medically unstable were not included in the sample, as these individuals were evacuated from the setting (i.e., Iraq); thus, data collection efforts were not possible. This yielded a clinical sample of patients with mild TBIs. Patients who completed all measures of interest were included in analyses. Study procedures were approved by the Brooke Army Medical Center Institutional Review Board, the US Army Medical Research and Materiel Command's Office of Research Protection, and the Multi- National Force-Iraq Institutional Official. The research was performed in compliance with the Code of Ethics of the World Medical Association (Declaration of Helsinki).

\section{Measures}

Traumatic Brain Injury (TBI). A mild TBI diagnosis was confirmed by a licensed clinical psychologist or physician during a clinical interview that assessed for the following criteria, which were obtained from Clinical Practice Guidelines issued by the U.S. Department of Veterans Affairs and U.S. Department of Defense (2016): a traumatically-induced structural injury and/or a physiological disruption of brain functioning caused by an external force. Immediately following the event, at least one of the following clinical signs must have developed or worsened: (a) period of loss of or decreased level of consciousness; (b) loss of memory for events immediately before or after the injury; (c) alteration in mental state at the time of the injury; (d) neurological deficits (transient or not); and (e) intracranial lesion.

Suicide Risk. Suicide risk was assessed utilizing the Suicidal Behaviors Questionnaire (SBQ-R; Osman et al., 2001). The SBQ-R is a 4-item self-report measure of the presence, 
severity, and frequency of lifetime suicidal ideation and attempts, and the likelihood of a future suicide attempt (e.g., How likely is it that you will attempt suicide someday?; $0=$ Never, $6=$ Very Likely). Overall scores range from 0 to 16, with higher scores reflecting greater suicide risk. The SBQ-R has acceptable-to-good internal consistency ( $\alpha=.76-.87$; Osman et al., 2001), as well as excellent test-retest reliability over two weeks $(r=.95)$ and strong convergent validity with other self-report scales of suicidality $(r=.69$ with the Scale for Suicidal Ideation; Cotton, Peters, \& Range, 1995). A systematic review has identified the SBQ-R as a paragon population-based suicide risk screening instrument (Batterham et al., 2015). In this sample, the internal reliability of the SBQ-R was acceptable $(\alpha=0.78)$.

Anger. Anger was assessed utilizing the mood scale module of the Automated Neuropsychological Assessment Metrics (ANAM; Johnson, Vincent, Johnson, Gilliland, \& Schlegel, 2008). The ANAM mood scale presents participants with adjectives describing eight different mood states (e.g., sleep, happiness, vigor, anger, depression). Respondents rate on a Likert-type scale the degree to which each adjective describes them at that moment in time. The anger subscale was utilized for the present study. The anger subscale has good convergent validity with the anger/hostility subscale of the Profile of Mood States $(r=0.65)$ and the anger/frustration subscale of the Dundee Stress State Questionnaire $(r=0.74)$, as well as excellent internal consistency $(\alpha=.91)$ and test-retest reliability ( $r=.78$; Johnson et al., 2008). Relevant to the present study, a corpus of research has utilized the ANAM scale among both civilian and military TBI populations (Ivins et al., 2009; Sours et al., 2015; Vincent et al., 2008).

Depression. Depression symptoms were assessed utilizing the Behavioral Health Measure-20 (BHM-20; Kopta \& Lowry, 2002). The 5-item depression subscale assesses the frequency over the preceding two weeks of low energy and motivation, not liking oneself, 
difficulties concentrating, sadness, and hopelessness. The scale ranges from 0 (almost always) to 4 (never), and the total score is obtained by calculating the mean of each item score. Given that higher scores reflect lower levels of depression symptoms, to facilitate ease in the interpretations of results, the scale was transformed for the current study such that higher scores indicated greater depression symptoms. The BHM-20 has demonstrated convergent validity with other validated depression and hopelessness scales across clinical and nonclinical samples (Blount et al., 2010). In this sample, the BHM-20 depression subscale demonstrated excellent internal consistency $(\alpha=.94)$.

To demonstrate internal replicability, the depression subscale from the ANAM was additionally utilized; notably, the items that comprise the depression subscale do not cross-load with the anger subscale (Johnson et al., 2008). The ANAM depression subscale has 6 items that are rated on a Likert-type scale reflecting intensity. It has excellent internal consistency $(\alpha=0.93)$ and convergent validity with the depression/dejection subscale of the Profile of Mood States $(r=0.71)$ and the Beck Depression Inventory ( $r=0.71$; Johnson et al., 2008).

\section{Data Analytic Strategy}

To test the hypotheses that the indirect effects of anger and depression symptoms would significantly account for the relationship between mild TBI and suicide risk, bootstrapped mediation analyses with 1,000 repetitions were conducted, consistent with guidelines recommended by Hayes (2013). A 95\% bias-corrected confidence interval (CI) around the point estimate of the indirect effect that does not cross zero indicates statistical significance. Given that we proposed a serial mediation model in which anger is hypothesized to precede depression, we constructed two separate models — one in which anger precedes depression symptoms, and one in 
which depression symptoms precede anger-in order to determine the importance of variable order. Analyses were conducted with SPSS version 20.0.0.

\section{Results}

\section{Descriptive Statistics}

Means, standard deviations, ranges, and zero-order Pearson $r$ correlation for all selfreport measures are presented in Table 2. Of note, $84.6 \%(126 / 149)$ of participants had a current mild TBI diagnosis. The modal number of previous TBIs was zero ( $\mathrm{n}=96 ; 64.4 \%)$, with 31 individuals reporting 1-2 previous TBIs, 12 individuals reporting 3-4 previous TBIs, and 10 individuals reporting 5 or more previous TBIs; the overall mean of previous TBIs was 1.0 $(\mathrm{SD}=2.1)$.

\section{Primary Analyses}

Figure 1 presents the path coefficients from the bootstrapped regression and mediation analyses for the effects of mild TBI on suicide risk through a sequential path of anger followed by depression symptoms. The overall regression model examining the relationship between mild TBI and suicide risk was statistically significant $\left(R^{2}=0.149, F[3,145]=8.489, p<0.001\right)$. Specifically, mild TBI significantly predicted anger $(B=11.845, S E=5.281, p=0.026)$, anger significantly predicted depression symptoms $(B=0.020, S E=0.002, p<0.001)$, and depression symptoms significantly predicted suicide risk $(B=0.788, S E=0.178, p<0.001)$. In this model, the direct effect of mild TBI on suicide risk was not statistically significant $(B=0.003, S E=0.339$, $p=0.993)$. The indirect effect of anger on the association between mild TBI and suicide risk was not statistically significant $(B=-0.092, S E=0.102$, bootstrapped $95 \% \mathrm{CI}=-0.397,0.027)$. The indirect effect of depression on the association between mild TBI and suicide risk was statistically significant $(B=0.464, S E=0.235$, bootstrapped $95 \% \mathrm{CI}=0.143,1.078)$. Importantly, in 
this model, the sequential indirect effects of anger and depression symptoms on the relationship between mild TBI and suicide risk were statistically significant $(B=0.189, S E=0.125$, bootstrapped $95 \% \mathrm{CI}=0.044,0.576$ ), indicating that a mild $\mathrm{TBI}$ is associated with increased anger, which is associated with increased depression symptoms, which in turn is linked to increased suicide risk. Given a significant indirect effect but no significant direct effect, this model indicates that the relationship between mild TBI and suicide risk is fully mediated by anger and depression symptoms.

Figure 2 presents the path coefficients from the bootstrapped regression and mediation analyses for the effects of mild TBI on suicide risk through a sequential path of depression symptoms followed by anger. In this model, in which the order of the sequential mediators was switched, the indirect effects of depression symptoms and anger on the relationship between mild TBI and suicide risk were no longer statistically significant $(B=-0.110, S E=0.110$, bootstrapped $95 \% \mathrm{CI}=-0.405,0.050)$. Thus, the order of the proposed mediators is meaningful, such that anger precedes depression symptoms in the mediational pathway between mild TBI and suicide risk.

\section{Sensitivity Analyses}

TBI Symptom Severity. Since the vast majority of patients had a mild TBI diagnosis $(84.6 \%)$, sensitivity analyses were performed utilizing the number of TBI symptoms as a proxy for TBI severity. Although past research has not yet firmly established if the number of TBI symptoms is indeed a reliable and valid proxy of TBI severity, research has demonstrated that TBI symptoms are correlated with disability indices at 3-month follow-up (Lundin et al., 2006). Moreover, individuals with a TBI report a significantly greater number of symptoms than individuals in other disability panels (e.g., spinal cord injury, HIV infection), suggesting that symptom counts are particularly relevant for TBI populations (Gordon et al., 2000). 
When a TBI symptom count is used as our predictor variable, the pattern of findings remained the same, such that the indirect effect for anger and depression on the association of mild TBI and suicide risk was statistically significant in the model in which anger preceded depression $(B=0.046, S E=0.024$, bootstrapped $95 \% \mathrm{CI}=0.012,0.115)$ but not in the model in which depression preceded anger $(B=-0.022, S E=0.022$, bootstrapped 95\% $\mathrm{CI}=-0.090,0.010)$.

Internal Replication. As noted, we endeavored to internally replicate the abovementioned findings utilizing a separate measure of depression symptoms that was administered to all participants (i.e., the ANAM depression subscale). In the analyses utilizing the mild TBI diagnosis variable, the indirect effect for anger and depression on the association of mild TBI and suicide risk was statistically significant in the model in which anger preceded depression $(B=0.230, S E=0.157$, bootstrapped $95 \% \mathrm{CI}=0.039,0.676)$ but not in the model in which depression preceded anger $(B=-0.145, S E=0.129$, bootstrapped 95\% CI=-0.496, 0.025). Similarly, in the analyses utilizing the TBI symptom severity index, the indirect effect for anger and depression on the association of TBI symptoms and suicide risk was statistically significant in the model in which anger preceded depression $(B=0.060, S E=0.036$, bootstrapped $95 \%$ $\mathrm{CI}=0.016,0.169)$ but not in the model in which depression preceded anger $(B=-0.035, S E=0.033$, bootstrapped $95 \% \mathrm{CI}=-0.132,0.005)$. Thus, utilizing a separate measure of depression symptoms, our findings that anger precedes depression in the pathway between mild TBI and suicide risk were internally replicated.

Future Likelihood of a Suicide Attempt. Item 4 of the SBQ-R assesses one's selfreported likelihood of making a future suicide attempt (i.e., How likely is it that you will attempt suicide someday?; $0=$ Never, $1=$ No chance at all, 2=Rather unlikely, 3=Unlikely, 4=Likely, 5=Rather likely, 6=Very likely). We constructed the abovementioned models utilizing SBQ-R 
Item 4 as the criterion variable, as this variable contains rich clinical data. The pattern of findings and statistical significance of all models remained unchanged (see Supplementary Table 1).

Past TBIs. Since an individual's experience with past TBIs may affect the phenomenology and associated clinical features of a recent TBI, including a greater number of mood symptoms (Dams-O'Connor et al., 2013), we additionally examined if the abovementioned models persisted while controlling for the number of previous TBIs a participant reported. The pattern and statistical significance of all models remained unchanged when entering the number of previous TBIs as a covariate into the mediation models (see Supplementary Table 1).

\section{Discussion}

Results from this study supported study hypotheses that, among a clinical sample of deployed military personnel, the indirect effects of anger and depression on the association of mild TBI and suicide risk are statistically significant. These findings persisted when examining suicide risk globally (i.e., the full SBQ-R), and the likelihood of making a future suicide attempt specifically (i.e., SBQ-R Item 4), as well as when accounting for the number of previous TBIs a participant reported experiencing.

What might account for the link between mild TBI, anger, depression, and suicide risk? Bryan and Clemans (2013) suggest that fluid vulnerability theory may be illuminative in this regard. Specifically, fluid vulnerability theory states that a culmination of predisposing vulnerabilities leads to increased sensitivity to emotional distress, which may thereby increase suicide risk (Rudd, 2006). Thus, in the context of the current study, a mild TBI is the predisposing vulnerability that may lead to increases in both anger and depression, which in turn serve to increase suicide risk. This is consistent with past research demonstrating that impaired emotion regulation following a TBI may account for increased anger (Aboulafia-Brakha et al., 
2015), as well as research linking TBI with psychiatric disorders and symptoms among military populations (Stéfan and Mathé, 2016). Importantly, although this suggests that a TBI functions as a predisposition for suicide risk, in the immediate wake of an injury, a TBI may have more dynamic properties, including triggering stress responses such as anger.

That mild TBIs lead to suicide risk via a sequential mediational pathway of anger and depression, and that this finding persists regardless of on one's previous history of a TBI, speaks to the potential importance of therapeutically impacting both anger and depression symptoms. Indeed, preliminary recommendations suggest that therapeutically targeting anger and other symptoms related to the loss of self may be efficacious in reducing suicide risk among individuals with a history of TBI (Brenner et al., 2009). Several interventions to reduce anger and depression among TBI patients have been studied, although it is acknowledged at the outset that studies examining suicidality as an outcome of these interventions are lacking. For example, in a pilot study testing an anger-specific cognitive behavior therapy (CBT) among individuals with an acquired brain injury, significant reductions were found in STAXI scores for the treatment group as compared to the control group (Medd and Tate, 2000). A separate trial found that, among individuals who had sustained a severe TBI, group CBT demonstrated efficacy in treating anger control problems as compared to the waitlist control (Walker et al., 2010).

In considering the clinical relevance of this study's findings, it is important to note the timing of the mild TBI assessment. The present study assessed for acute, mild TBIs rather than chronic TBI sequelae. That we found associations between acute, mild TBI and suicidality is particularly noteworthy, as this may signal to clinicians that suicidality may develop in the nearterm post-TBI injury and repeated trauma may not be necessary for the emergence of increased suicide risk. This is important because existing research in suicidology has largely focused on 
chronic, long-term risk factors rather than acute risk factors (Klonsky et al., 2016). Further, although we assessed suicide risk broadly (i.e., suicidal ideation and past suicide attempts), the significant findings utilizing Item 4 of the SBQ-R, which assesses the future likelihood of making a suicide attempt, as the criterion variable might be most temporally relevant given the acuity of the mild TBI diagnoses in this sample.

\section{Limitations and Future Directions}

Several methodological limitations should be noted, including the utilization of crosssectional data, which limits our ability to establish causality. Indeed, although we have tested a mediation model utilizing gold standard approaches (Hayes, 2013), the nature of our data do not allow for causal inferences. Further, while self-reported anger and depression captures the subjective experiences of these conditions, behavioral observation of anger-related symptoms can provide incremental information (cf. Ribeiro, Bender, Selby, Hames, \& Joiner, 2011). Relatedly, the depression and anger variables reflected symptoms rather than a diagnosis of a depressive disorder or an anger-related disorder (e.g., IED), respectively. Moreover, our outcome variable was limited to suicide risk, which reflects an index comprised of current suicidal ideation, past history of suicide attempts, and future likelihood of suicide attempt. While this index of suicidality has marshaled strong empirical support (Batterham et al., 2015), our conclusions may not generalize to actual future suicide attempts or suicide deaths. Similarly, the mean and standard deviation of the index of suicide risk (i.e., 3.48 and 1.50, respectively, on the SBQ-R) suggests that the majority of participants did not meet the previously established cutoff indicating severe suicide risk (i.e., 8, validated among psychiatric inpatients; Osman et al., 2001). The sample did indeed report a nonzero level of suicide risk, indicating clinical relevance; however, future research should test study hypotheses among a more clinically severe sample. 
Moreover, nearly two-thirds of participants did not report a prior TBI history; thus, although the pattern of our results remained the same when controlling for previous TBIs, we may have been underpowered for these analyses. As such, findings may not generalize to individuals who have experienced multiple TBIs.

Future research should examine this study's conjectures among individuals with a more chronic/repeated history of TBIs. Future research should also systematically examine the various levels of TBI severity — mild, moderate, severe — and to determine if anger has a differential role in the association of TBI and suicide risk along the severity gradient. Relatedly, it is important for future research to quantify the degree of cognitive impairment experienced by participants. Finally, it is important to test if therapeutically targeting anger and depression symptoms actually serves to decrease suicide risk among military personnel who have sustained a TBI. To address the abovementioned limitations, future research would benefit from a prospective design using multimodal methodologies (e.g., self-report of TBI symptoms and functional impact, behavioral observations) among a clinical sample of military personnel. 


\section{References}

Aboulafia-Brakha, T., Allain, P., Ptak, R., 2015. Emotion regulation after traumatic brain injury: Distinct patterns of sympathetic activity during anger expression and recognition. J. Head Trauma Rehabil. 1. doi:10.1097/HTR.0000000000000171

Alderman, N., 2003. Contemporary approaches to the management of irritability and aggression following traumatic brain injury. Neuropsychol. Rehabil. 13, 211-240. doi:10.1080/09602010244000327

American Psychiatric Association, 2013. Diagnostic and Statistical Manual of Mental Disorders, 5th Edition: DSM-5. American Psychiatric Association, Washington, DC.

Bahraini, N.H., Simpson, G.K., Brenner, L.A., Hoffberg, A.S., Schneider, A.L., 2013. Suicidal ideation and behaviours after traumatic brain injury: A systematic review. Brain Impair. 14, 92-112. doi:10.1017/BrImp.2013.11

Bailie, J.M., Cole, W.R., Ivins, B., Boyd, C., Lewis, S., Neff, J., Schwab, K., 2015. The experience, expression, and control of anger following traumatic brain injury in a military sample. J. Head Trauma Rehabil. 30, 12-20. doi:10.1097/HTR.0000000000000024

Batterham, P.J., Ftanou, M., Pirkis, J., Brewer, J.L., Mackinnon, A.J., Beautrais, A., FairweatherSchmidt, A.K., Christensen, H., 2015. A systematic review and evaluation of measures for suicidal ideation and behaviors in population-based research. Psychol. Assess. 27, 501-512. doi:10.1037/pas0000053

Blount, T.H., Bryan, C.J., Kanzler, K.E., Morrow, C.E., Corso, K.A., Corso, M.L., 2010. Psychometric properties of the BHM-20 in military samples, in: Association for Behavioral and Cognitive Therapy. San Francisco, CA.

Brenner, L.A., Bahraini, N., Homaifar, B.Y., Monteith, L.L., Nagamoto, H., Dorsey-Holliman, B., Forster, J.E., 2015. Executive functioning and suicidal behavior among veterans with and without a history of traumatic brain injury. Arch. Phys. Med. Rehabil. 96, 1411-1418. doi:10.1016/j.apmr.2015.04.010

Brenner, L.A., Homaifar, B.Y., Adler, L.E., Wolfman, J.H., Kemp, J., 2009. Suicidality and veterans with a history of traumatic brain injury: Precipitating events, protective factors, and prevention strategies. Rehabil. Psychol. 54, 390-397. doi:10.1037/a0017802

Brenner, L.A., Homaifar, B.Y., Olson-Madden, J.H., Nagamoto, H.T., Huggins, J., Schneider, A.L., Forster, J.E., Matarazzo, B., Corrigan, J.D., 2013. Prevalence and screening of traumatic brain injury among veterans seeking mental health services. J. Head Trauma Rehabil. 28, 21-30. doi:10.1097/HTR.0b013e31827df0b5

Brenner, L.A., Ignacio, R. V, Blow, F.C., 2011. Suicide and traumatic brain injury among individuals seeking Veterans Health Administration services. J. Head Trauma Rehabil. 26, 257-264. doi:10.1097/HTR.0b013e31821fdb6e

Brown, G.K., Beck, A.T., Steer, R.A., Grisham, J.R., 2000. Risk Factors for Suicide in Psychiatric Outpatients: A 20-Year Prospective Study. J. Consult. Clin. Psychol. 68, 371377. doi:10.1037/0022-006X.68.3.371

Bryan, C.J., Clemans, T.A., 2013. Repetitive traumatic brain injury, psychological symptoms, and suicide risk in a clinical sample of deployed military personnel. JAMA Psychiatry 70, 686-691. doi:10.1001/jamapsychiatry.2013.1093

Cavanagh, J.T., Carson, A.J., Sharpe, M., Lawrie, S.M., 2003. Psychological autopsy studies of suicide: A systematic review. Psychol. Med. 33, 395-405.

doi:10.1017/S0033291702006943 
Centers for Disease Control and Prevention [CDC], 2016. Leading Causes of Death Reports, National and Regional, 1999 - 2014.

Chu, C., Klein, K.M., Buchman-Schmitt, J.M., Hom, M.A., Hagan, C.R., Joiner, T.E., 2015. Routinized assessment of suicide risk in clinical practice: An empirically informed update. J. Clin. Psychol. 71, 1186-1200. doi:10.1002/jclp.22210

Cotton, C.R., Peters, D.K., Range, L.M., 1995. Psychometric properties of the suicidal behaviors questionnaire. Death Stud. 19, 391-397. doi:10.1080/07481189508252740

Dams-O’Connor, K., Spielman, L., Singh, A., Gordon, W.A., Lingsma, H.F., Maas, A.I.R., Manley, G.T., Mukherjee, P., Okonkwo, D.O., Puccio, A.M., Schnyer, D.M., Valadka, A.B., Yue, J.K., Yuh, E.L., The TRACK-TBI Investigators, 2013. The Impact of Previous Traumatic Brain Injury on Health and Functioning: A TRACK-TBI Study. J. Neurotrauma 30, 2014-2020. doi:10.1089/neu.2013.3049

Defense Suicide Prevention Office, 2014. Fiscal Year 2013 Annual Report. Arlington, VA. Department of Veterans Affairs, Department of Defense, 2016. VA/DoD Clinical Practice Guideline for the Management of Concussion-Mild Traumatic Brain Injury.

Fava, M., Rosenbaum, J.F., Pava, J.A., McCarthy, M.K., Steingard, R.J., Bouffides, E., 1993. Anger attacks in unipolar depression, Part 1: Clinical correlates and response to fluoxetine treatment. Am. J. Psychiatry 150, 1158-1163. doi:10.1176/ajp.150.8.1158

Gordon, W.A., Haddad, L., Brown, M., Hibbard, M.R., Sliwinskia, M., 2000. The sensitivity and specificity of self-reported symptoms in individuals with traumatic brain injury. Brain Inj. 14, 21-33. doi:10.1080/026990500120907

Gradus, J.L., Wisco, B.E., Luciano, M.T., Iverson, K.M., Marx, B.P., Street, A.E., 2015. Traumatic brain injury and suicidal ideation among U.S. Operation Enduring Freedom and Operation Iraqi Freedom veterans. J. Trauma. Stress 28, 361-365. doi:10.1002/jts.22021

Gutierrez, P.M., Brenner, L.A., Huggins, J.A., 2008. A preliminary investigation of suicidality in psychiatrically hospitalized veterans with traumatic brain injury. Arch. Suicide Res. 12, 336-343. doi:10.1080/13811110802324961

Hawkins, K.A., Cougle, J.R., 2013. A test of the unique and interactive roles of anger experience and expression in suicidality. J. Nerv. Ment. Dis. 201, 959-963. doi:10.1097/NMD.0000000000000041

Hayes, A.F., 2013. Introduction to mediation, moderation, and conditional process analysis: A regression-based approach. Guilford Press, New York, NY.

Hoge, C.W., Castro, C.A., 2012. Preventing suicides in US service members and veterans: Concerns after a decade of war. JAMA 308, 671-2. doi:10.1001/jama.2012.9955

Ivins, B.J., Kane, R., Schwab, K.A., 2009. Performance on the Automated Neuropsychological Assessment Metrics in a nonclinical sample of soldiers screened for mild TBI after returning from Iraq and Afghanistan. J. Head Trauma Rehabil. 24, 24-31. doi:10.1097/HTR.0b013e3181957042

Johnson, D.R., Vincent, A.S., Johnson, A.E., Gilliland, K., Schlegel, R.E., 2008. Reliability and construct validity of the Automated Neuropsychological Assessment Metrics (ANAM) mood scale. Arch. Clin. Neuropsychol. 23, 73-85. doi:10.1016/j.acn.2007.10.001

Joiner, T.E., 2005. Why people die by suicide. Harvard University Press, Cambridge, MA.

Joiner, T.E., Stanley, I.H., n.d. Can the phenomenology of a suicidal crisis be usefully understood as a suite of antipredator defensive reactions? Psychiatry Interpers. Biol. Process.

Kemp, J., Bossarte, R., 2013. Suicide Data Report, 2012. Washington, DC. 
Kim, S.H., Manes, F., Kosier, T., Baruah, S., Robinson, R.G., 1999. Irritability following traumatic brain injury. J. Nerv. Ment. Dis. 187, 327-335. doi:10.1097/00005053199906000-00001

Klonsky, E.D., May, A.M., Saffer, B.Y., 2016. Suicide, suicide attempts, and suicidal ideation. Annu. Rev. Clin. Psychol. 12, annurev-clinpsy-021815-093204. doi:10.1146/annurevclinpsy-021815-093204

Koh, K.B., Kim, C.H., Park, J.K., 2002. Predominance of anger in depressive disorders compared with anxiety disorders and somatoform disorders. J. Clin. Psychiatry 63, 486492. doi:10.4088/JCP.v63n0604

Koponen, S., Taiminen, T., Portin, R., Himanen, L., Isoniemi, H., Heinonen, H., Hinkka, S., Tenovuo, O., 2002. Axis I and II psychiatric disorders after traumatic brain injury: A 30year follow-up study. Am. J. Psychiatry 159, 1315-1321. doi:10.1176/appi.ajp.159.8.1315

Kopta, S.L., Lowry, J.L., 2002. Psychometric evaluation of the Behavioral Health Questionnaire20: a brief, comprehensive instrument for assessing mental health. Psychother. Res. 12, 413-426.

Kuehn, B.M., 2009. Soldier suicide rates continue to rise: military, scientists work to stem the tide. JAMA 301, 1111, 1113. doi:10.1001/jama.2009.342

Lundin, A., de Boussard, C., Edman, G., Borg, J., 2006. Symptoms and disability until 3 months after mild TBI. Brain Inj. 20, 799-806. doi:10.1080/02699050600744327

Luutonen, S., 2007. Anger and depression-Theoretical and clinical considerations. Nord. J. Psychiatry 61, 246-251. doi:10.1080/08039480701414890

Medd, J., Tate, R.L., 2000. Evaluation of an anger management therapy programme following acquired brain injury: A preliminary study. Neuropsychol. Rehabil. 10, 185-201. doi:10.1080/096020100389246

Minkoff, K., Bergman, E., Beck, A.T., Beck, R., 1973. Hopelessness, Depression, and Attempted Suicide. Am. J. Psychiatry 130, 455-459.

Nock, M.K., Stein, M.B., Heeringa, S.G., Ursano, R.J., Colpe, L.J., Fullerton, C.S., Hwang, I., Naifeh, J.A., Sampson, N.A., Schoenbaum, M., Zaslavsky, A.M., Kessler, R.C., 2014. Prevalence and correlates of suicidal behavior among soldiers: Results from the Army Study to Assess Risk and Resilience in Servicemembers (Army STARRS). JAMA Psychiatry 71, 514-22. doi:10.1001/jamapsychiatry.2014.30

Osman, A., Bagge, C.L., Gutierrez, P.M., Konick, L.C., Kopper, B.A., Barrios, F.X., 2001. The Suicidal Behaviors Questionnaire-Revised (SBQ-R): Validation with clinical and nonclinical samples. Assessment 8, 443-454. doi:10.1177/107319110100800409

Potegal, M., Stemmler, G., 2010. Cross-disciplinary views of anger: Consensus and controversy, in: International Handbook of Anger. Springer New York, New York, NY, pp. 3-7. doi:10.1007/978-0-387-89676-2_1

Ramchand, R., Acosta, J., Burns, R.M., Jaycox, L.H., Pernin, C.G., 2011. The War Within: Preventing Suicide in the US Military. Santa Monica, CA.

Rao, V., Rosenberg, P., Bertrand, M., Salehinia, S., Spiro, J., Vaishnavi, S., Rastogi, P., Noll, K., Schretlen, D.J., Brandt, J., Cornwell, E., Makley, M., Miles, Q.S., 2009. Aggression after traumatic brain injury: Prevalence and correlates. J. Neuropsychiatr. 21, 420-429. doi:10.1176/appi.neuropsych.21.4.420

Ribeiro, J.D., Bender, T.W., Selby, E.A., Hames, J.L., Joiner, T.E., 2011. Development and validation of a brief self-report measure of agitation: The Brief Agitation Measure. J. Pers. Assess. 93, 597-604. doi:10.1080/00223891.2011.608758 
Rudd, M.D., 2006. The assessment and management of suicidality. Professional Resource Press, Sarasota, FL.

Skopp, N.A., Trofimovich, L., Grimes, J., Oetjen-Gerdes, L., Gahm, G.A., 2012. Relations between suicide and traumatic brain injury, psychiatric diagnoses, and relationship problems, active component, U.S. Armed Forces, 2001-2009. MSMR 19, 7-11.

Sours, C., Rosenberg, J., Kane, R., Roys, S., Zhuo, J., Shanmuganathan, K., Gullapalli, R.P., 2015. Associations between interhemispheric functional connectivity and the Automated Neuropsychological Assessment Metrics (ANAM) in civilian mild TBI. Brain Imaging Behav. 9, 190-203. doi:10.1007/s11682-014-9295-y

Stéfan, A., Mathé, J.-F., 2016. What are the disruptive symptoms of behavioral disorders after traumatic brain injury? A systematic review leading to recommendations for good practices. Ann. Phys. Rehabil. Med. doi:10.1016/j.rehab.2015.11.002

Tanielian, T., Jaycox, L.H., 2008. Invisible Wounds of War: Psychological and Cognitive Injuries, Their Consequences, and Services to Assist Recovery, RAND Corporation. RAND Corporation, Santa Monica, CA. doi:10.1176/appi.ps.60.2.273

Teasdale, T.W., Engberg, A.W., 2001. Suicide after traumatic brain injury: a population study. J. Neurol. Neurosurg. Psychiatry 71, 436-440.

Terrio, H., Brenner, L.A., Ivins, B.J., Cho, J.M., Helmick, K., Schwab, K., Scally, K., Bretthauer, R., Warden, D., 2009. Traumatic brain injury screening: Preliminary findings in a US Army Brigade Combat Team. J. Head Trauma Rehabil. 24, 14-23. doi:10.1097/HTR.0b013e31819581d8

US Department of Health and Human Services Office of the Surgeon General and National Action Alliance for Suicide Prevention, 2012. National Strategy for Suicide Prevention 2012: Goals and Objectives for Action. Washington, DC.

Van Orden, K.A., Witte, T.K., Cukrowicz, K.C., Braithwaite, S.R., Selby, E.A., Joiner, T.E., 2010. The interpersonal theory of suicide. Psychol. Rev. 117, 575-600. doi:10.1037/a0018697

Vincent, A.S., Bleiberg, J., Yan, S., Ivins, B., Reeves, D.L., Schwab, K., Gilliland, K., Schlegel, R., Warden, D., 2008. Reference data from the Automated Neuropsychological Assessment Metrics for use in traumatic brain injury in an active duty military sample. Mil. Med. 173, 836-852. doi:10.7205/MILMED.173.9.836

Walker, A.J., Nott, M.T., Doyle, M., Onus, M., McCarthy, K., Baguley, I.J., 2010. Effectiveness of a group anger management programme after severe traumatic brain injury. Brain Inj. 24, 517-524. doi:10.3109/02699051003601721 


\section{Acknowledgements}

This research was supported in part by the Military Suicide Research Consortium, an effort supported by the Office of the Assistant Secretary of Defense for Health Affairs under Award No. (W81XWH-10-2-0181). Opinions, interpretations, conclusions and recommendations are those of the authors and are not necessarily endorsed by the Military Suicide Research Consortium or the Department of Defense. 


\section{Figures}

Figure 1: Anger and depression symptoms sequentially mediating the relationship between mild traumatic brain injury and suicide risk $(\mathrm{N}=149)$

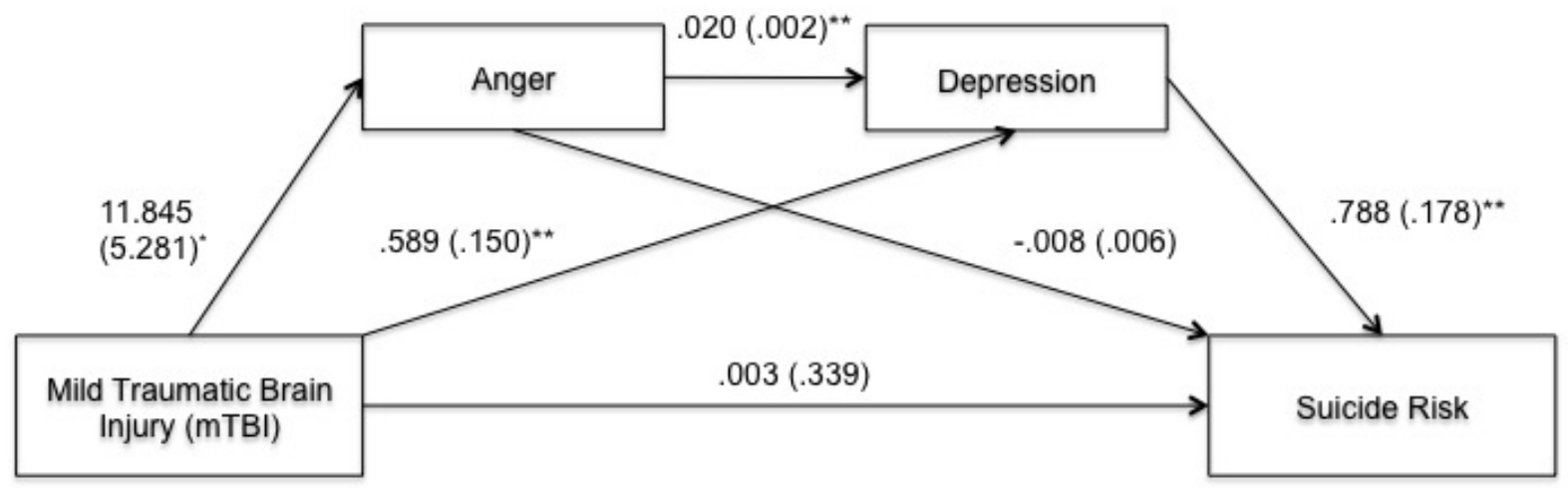

Note. The sequential indirect effects of anger and depression symptoms on the association between mild traumatic brain injury $(\mathrm{mTBI})$ and suicide risk were statistically significant $(B=$ $0.189, S E=0.125$, bootstrapped $95 \% \mathrm{CI}=0.044,0.576) . * p<.05, * * p<.01$.

Figure 2: Depression symptoms and anger sequentially mediating the relationship between mild traumatic brain injury and suicide risk $(\mathrm{N}=149)$

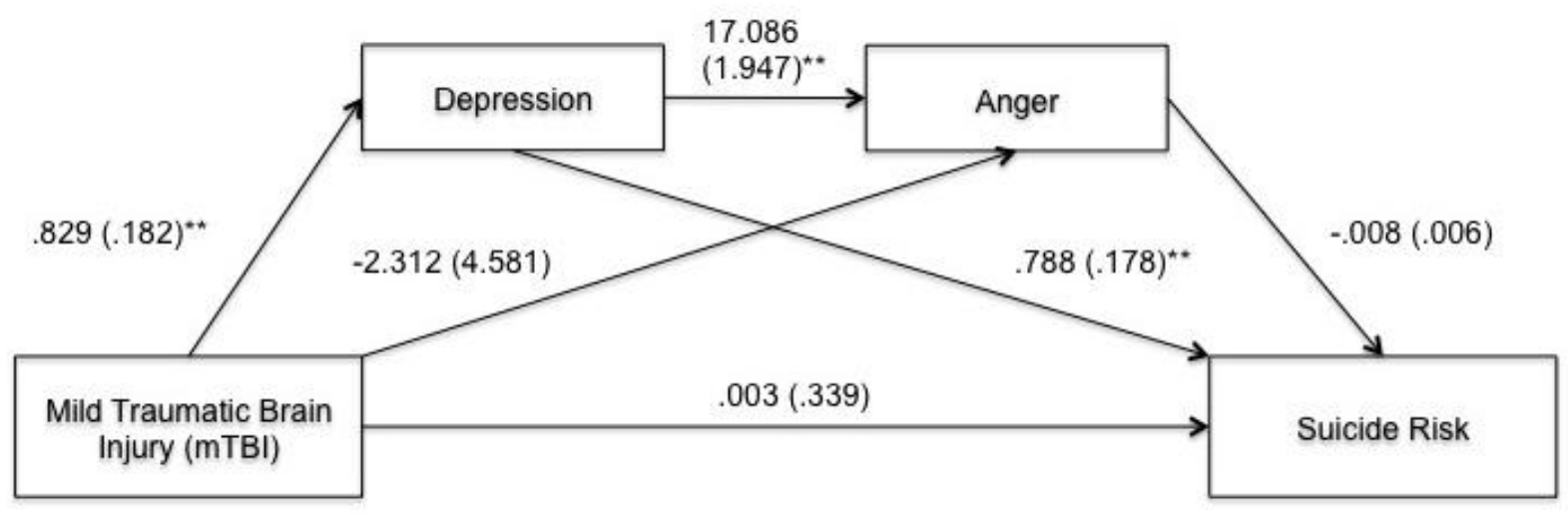

Note. The sequential indirect effects of depression symptoms and anger on the association between mild traumatic brain injury (mTBI) and suicide risk were not statistically significant ( $B$ $=-0.110, S E=.110$, bootstrapped $95 \% \mathrm{CI}=-0.405,0.050) .{ }^{*} p<.05, * * p<.01$. 


\section{Tables}

Table 1: Participant Characteristics $(\mathrm{N}=149)$

\begin{tabular}{lc}
\hline Characteristic & Value \\
\hline Sex, No. (Valid \%) & $138(92.6)$ \\
Male & $11(7.4)$ \\
Female & \\
Race, No. (Valid \%) & $105(71.4)$ \\
White & $23(15.6)$ \\
African American & $14(9.5)$ \\
Hispanic/Latino & $4(2.7)$ \\
Asian/Pacific Islander & $1(0.7)$ \\
Other & $2(--)$ \\
Missing & \\
Branch, No. (Valid \%) & $119(79.9)$ \\
Army & $20(13.4)$ \\
Air Force & $8(5.4)$ \\
Marines & $2(1.3)$ \\
Civilian & \\
Status, No. (Valid \%) & $71(53.8)$ \\
Active Duty & $54(40.9)$ \\
National Guard & $5(3.8)$ \\
Reserves & $2(1.5)$ \\
Civilian & $17(--)$ \\
Missing & \\
Rank, No. (Valid \%) & $82(55.8)$ \\
E1-E4 & $49(33.3)$ \\
E5-E6 & $8(5.4)$ \\
E7-E9 & $1(0.7)$ \\
Warrant Officer & $7(4.8)$ \\
Officer & $2(--)$ \\
Missing & $27.9(7.2)^{\mathrm{a}}$ \\
Age, mean (SD), y & $6.7(5.4)^{\mathrm{b}}$ \\
Time in military, mean (SD), y & $0.8(1.1)^{\mathrm{c}}$ \\
Prior deployments, mean (SD) & \\
${ }^{\mathrm{a}} \mathrm{N}=147{ }^{\mathrm{b}}$ N = 136; ${ }^{\mathrm{N}} \mathrm{N}=148$. & \\
&
\end{tabular}


Table 2: Means, Standard Deviations, and Intercorrelations of Measures

\begin{tabular}{lccccc}
\hline & $\mathbf{1}$ & $\mathbf{2}$ & $\mathbf{3}$ & $\mathbf{4}$ & $\mathbf{5}$ \\
\hline 1. mTBI & 1 & & & & \\
2. SBQ-R Suicide Risk & $.136^{\mathrm{a}}$ & 1 & & & \\
3. ANAM Anger & $.182^{*}$ & $.149^{\mathrm{a}}$ & 1 & & \\
4. ANAM Depression & $.280^{* *}$ & $.341^{* *}$ & $.702^{* *}$ & 1 & \\
5. BEH-20 Depression & $.352^{* *}$ & $.374^{* *}$ & $.605^{* *}$ & $.838^{* *}$ & 1 \\
M & 0.85 & 3.48 & 23.06 & 17.67 & 0.90 \\
SD & 0.36 & 1.50 & 23.61 & 22.20 & 0.85 \\
Minimum & 0 & 3 & 0 & 0 & 0 \\
Maximum & 1 & 16 & 94 & 86 & 3.40 \\
\hline
\end{tabular}

${ }^{*} p<.05,{ }^{* *} p<.01,{ }^{\mathrm{a}} p<.10 ;$ ANAM, Automated Neuropsychological

Assessment Metrics; BEH-20, Behavioral Health Measure-20;. mTBI, mild traumatic brain injury; SBQ-R, Suicidal Behaviors Questionnaire-Revised; TBI, traumatic brain injury. mTBI is a dichotomous variable that is included in the correlation matrix for descriptive purposes. 